\title{
Total Arterial Coronary Revascularization with Aorto-bifemoral Bipopliteal Bypass - A Case Report
}

\author{
Nuruddin Mohammod Zahangir, Syed Tanvir Ahmad, Firoz Ahmed, Md. Mainul Kabir, Tamjid \\ Mohammad Najmus Sakib Khan, Nazmul Hossain, Niaz Ahmed, Madhava Janardhan Naik \\ Department of Cardiothoracic Surgery, Apollo Hospitals, Dhaka, Bangladesh
}

\author{
Key Words : \\ Total arterial, \\ coronary \\ revascularization, \\ Aorto-bifemoral \\ bypass,Bifemoro- \\ bipopliteal \\ bypass.
}

\begin{abstract}
:
The management of combined Coronary Artery Disease and Peripheral Vascular Disease is a challenge and brings with it numerous clinical dilemmas. The 56 year old gentleman presented to our department with significant triple vessel disease with occluded lower end of aorta just before bifurcation and occluded bilateral superficial femoral arteries. CABG with aorto-femoral and femoro popliteal bypass was done on the same setting. On $12^{\text {th }}$ postoperative day he was discharged from hospital in good general condition. Follow up after 6 months of operation the patient is doing well and free of chest pain and claudication pain.
\end{abstract}

(Cardiovasc. j. 2015; 7(2): 159-161)

\section{Introduction:}

Aorto-bifemoral bipopliteal bypass with total arterial coronary artery bypass is a rare combined single sitting operation in Bangladesh. Coronary artery disease $(\mathrm{CAD})$ and peripheral vascular disease (PVD) remain highly prevalent in the population due to population ageing, smoking, diabetes, unhealthy lifestyles, and the epidemic of obesity, and frequently coexist. The management of combined $\mathrm{CAD}$ and PVD is a challenge and brings with it numerous clinical dilemmas. This case report highlights issues related to the peri-operative evaluation and management of patients going to Aorto-bifemoral bipopliteal bypass grafting along with total arterial coronary artery bypass surgery.

\section{Case Report:}

The 56 year old gentleman presented to the department of Cardiology for intermittent claudication (Bilateral) for 3 months for which he underwent investigation with a coronary and peripheral angiogram. The patient was diabetic (good control with insulin), renal function, pulmonary function was normal with stable cardiac function (EF was 60\%). Coronary and peripheral angiogram showed significant triple vessel disease with occluded lower end of aorta just before bifurcation and occluded bilateral superficial femoral arteries. As one condition precluded the treatment of the other, a combined procedure was thought of and discussed with the patient. A CT Angiogram (Fig-1) was performed to assess feasibility of revascularization and also for planning the procedure. The risks and benefits of a combined procedure (as it involved an incision extending from the top of the chest to the symphysis pubis and along the leg to the ankles) was discussed with the patient; which he understood and took 3 weeks to decide. A prolonged recovery time was also discussed and understood.

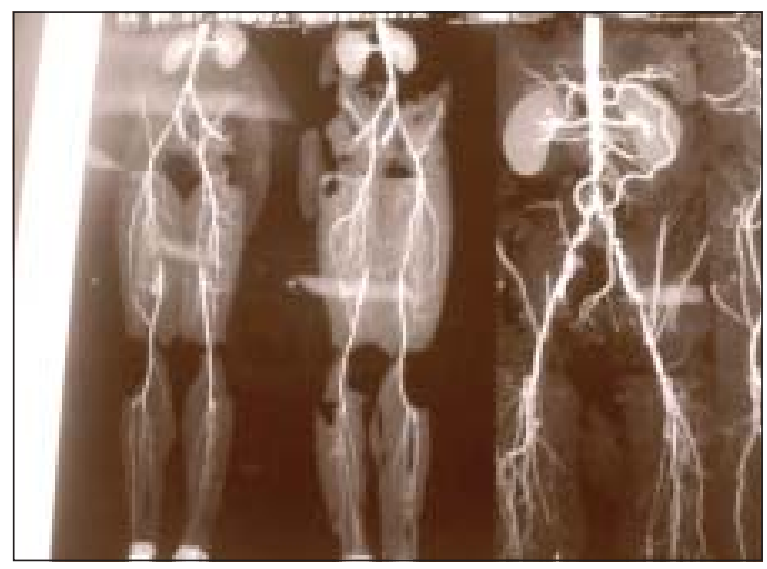

Fig.-1: CT angiogram showing significant stenosis in abdominal aorta and ilateral superficial femoral artery.

Address of Correspondence: Dr. Nuruddin Mohammod Zahangir, Dept. of Cardio Thoracic Surgery, Apollo Hospitals, Dhaka, Bangladesh.E-mail: nmzaha@yahoo.com 
Operation was done on 11.03.14. The Left and Right Internal Mammary Arteries (LIMA \& RIMA) were harvested in skeletonized manner. The free RIMA was then anastomosed with the LIMA to make LIMA-RIMA Y (Fig 2 ). During harvesting of internal mammary arteries, exposure of the Common Femoral arteries, Superficial Femoral arteries, Deep Femoral arteries and Popliteal arteries on both sides was done to assess suitability of grafting.

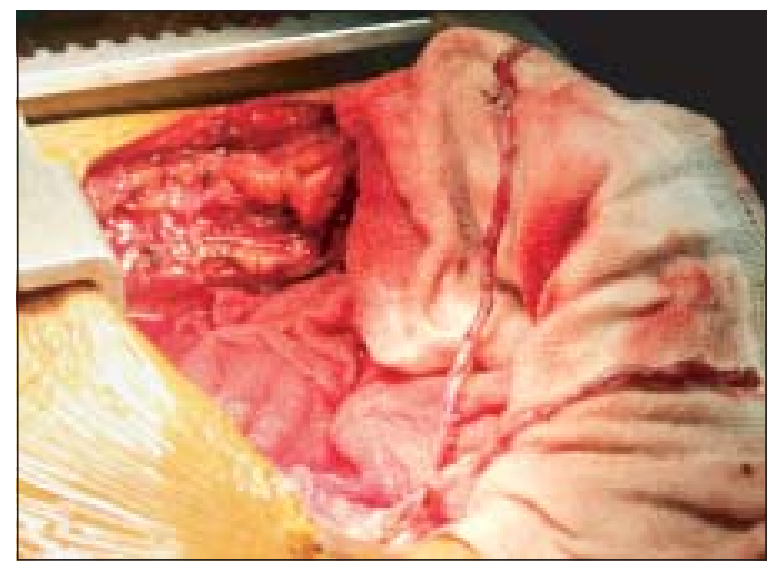

Fig.-2: LIMA-RIMA Y Graft for CABG.

Cardio Pulmonary Bypass (CPB) was then established and coronary artery bypass grafts performed in the following way. Left Internal Mammary Artery (LIMA) was anastomosed to the Left Anterior Descending artery. Right Internal Mammary Artery (RIMA) was anastomosed to Posterior Descending artery, $1^{\text {st }}$ and $2^{\text {nd }}$ Obtuse Marginal arteries in a sequential manner. Aortic cross-clamp was released and the heart started beating spontaneously.

While the patient continued on Cardio Pulmonary Bypass, the abdomen was opened by midline incision. The infrarenal aorta exposed and was found to be severely diseased. Urologist was involved in the abdminal part of surgery and exposure of the infra-renal aorta. A Vascular Clamp was applied infrarenally (Fig-3), Aortotomy done (Fig 4) and limited endarterectomy performed (Fig5) to gain site for top-end anastomosis. An end-toside anastomosis was done with upper end of PTFE synthetic Y-graft $(14 / 7 \mathrm{~mm})$ to the infra renal aorta (Fig-6) and the Clamp released. Good flow noted in both limbs of the graft. The patient was then slowly weaned off from Cardio Pulmonary Bypass without difficulty.
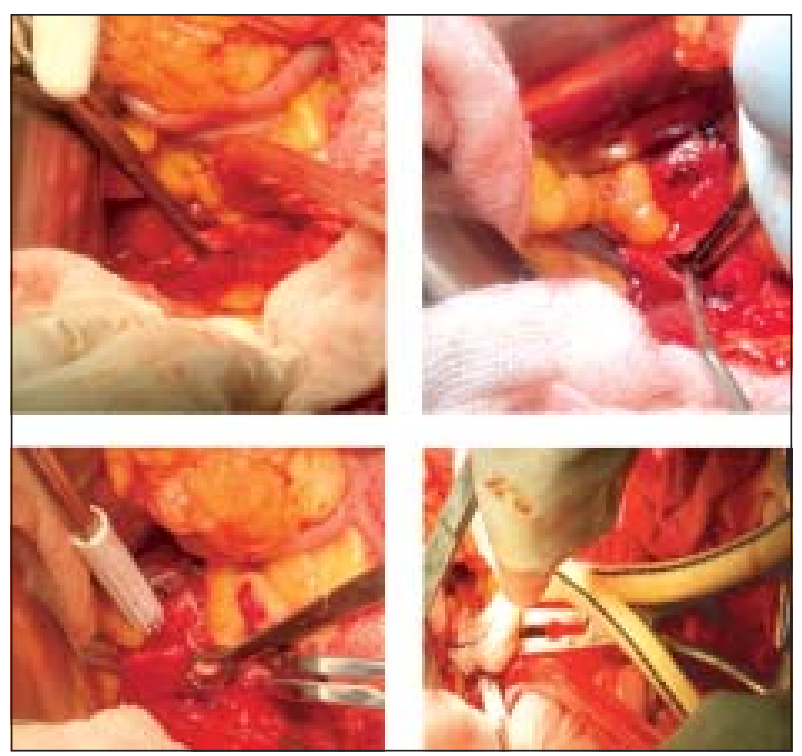

Fig 3-6: Different stes of Aorto femoral bypass. (Fig 3- Infrarenal aorta clamped, Fig 4-Aortotomy, Fig 5- Endarterectomy, Fig 6-: Synthetic Y graft anaestomosis to aorta).

The distal two limbs of the Y graft were passed to the groin through retroperitoneal tunnels and anastomosed end-to-side with the common femoral arteries. Saphenous vein was interposed between common femoral and popliteal arteries bilaterally through subfascial tunnels in both thigh(Fig-7).

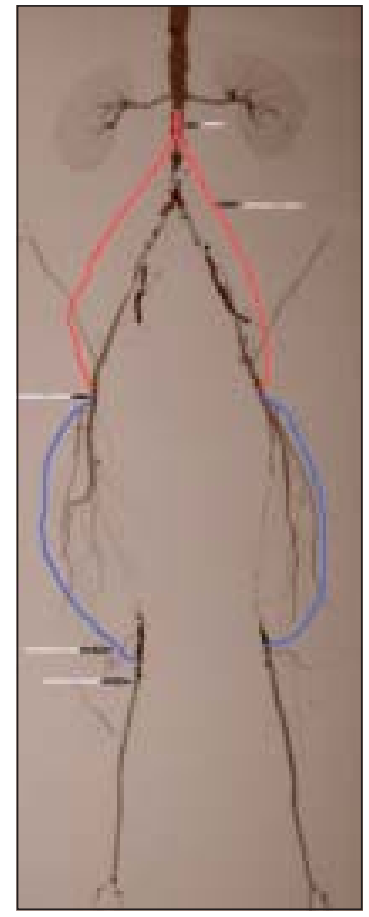

Fig-7 : Graphical presentation of anastomosis done in the lowerlimbs. 
Extra Corporeal Circulation Time was 246 minutes; cross clamp time was 125 minutes. The patient had a minor stroke on 2 nd postoperative day from which he recovered well. He was discharged from ICU on 7 th postoperative day soon. On $12^{\text {th }}$ postoperative day he was discharged from hospital in good general condition.

\section{Discussion:}

A retrospective review was conducted at Groote Schuur Hospital, Cape Town, between January 1998 and December 2001. During this period, 65 patients underwent femoro-distal bypass. The median age of the patients in this study was 62 years. Twenty-eight patients (49\%) were diabetic. An overall 2- year mortality of $19.2 \%$ was recorded. Reversed saphenous vein graft (RSVG) was used in 29 legs $(50.9 \%) .{ }^{1}$ Our patient was 56 years old gentleman and Femoro bi-popliteal bypass was done by Reversed saphenous vein graft. CT angiography appears consistent and accurate in the assessment of patients with peripheral arterial occlusive disease. ${ }^{2}$

Association of extracorporal assisted coronary bypass with peripheral vascular surgery has already place common in the therapeutic arsenal. Synchronous coronary bypass graft and aortoprofundal bypass in one single setting were performed. We believe that this type of synchronous procedure, minimizing surgical aggression, could be effective in selected high risk patients. ${ }^{3}$ We performed total arterial bypass graft with aorto-bifemoral and bilateral femoro-popliteal bypass graft.

Patients with the combination of coronary artery disease and peripheral vascular disease are frequently encountered in clinical practice. They tend to have multiple coronary risk factors and manifest widespread vascular disease. Clinical decision making is complex and challenging. CABG in appropriately selected patients has important short- and long-term benefits, albeit at a higher immediate risk. ${ }^{4}$

ESC(European society of cardiology) Class 1 recommendations for management of patients with peripheral arterial disease and concomitant Coronary artery disease- In patients with unstable Coronary artery disease, vascular surgery should be postponed and CAD treated first, except when vascular surgery cannot be delayed due to a life- or limb-threatening condition. In our case our patient presented with threatening limb ischemia with Coronary artery disease.

Use of the bilateral Internal Mammary Arteries offers the possibility of constructing various configurations, making total arterial myocardial revascularization possible with a minimum number of arterial conduits. We used here skeletonized LIMA-RIMA Y graft. Patients who received bilateral Internal Mammary Arteries grafts had improved early and late outcomes and decreased risk of death, reoperation, and angioplasty ${ }^{5}$. Close collaboration between cardiologists, cardio-vascular surgeons, urologist and anaesthetists is the key to successful management of such patients ${ }^{6}$.

\section{Conclusion:}

Combined CAD and PVD are never easy and are in general extremely complex. For getting a good result a close collaboration between specialists from many different areas of expertise cardiology, cardio-vascular surgery, neurology, nephrology,radiology is needed.

\section{Conflict of Interest - None.}

\section{References:}

1. Mwipatayi BP., Jeffery PC.,Motale, P. et al. Femoro-distal bypass surgery at Groote Schuur hospital-4-year retrospective stud. S Afr J Surg 2004 Aug; 42(3):81-85.

2. Catalano C1, Fraioli F, Laghi A et al. Infrarenal aortic and lower-extremity arterial disease: diagnostic performance of multi-detector row CT angiography. Radiology 2004; 231(2): 555-563.

3. Alexandrescu V, Bergeron P, Perrin R, Khanoyan P, Rosario R. Combined minimally invasive surgery for coronary bypass and abdominal aortic occlusion. Cardiovasc Surg 2000 Oct;8(6): 507-509.

4. Charanjit S R, Kim A E, Mary C M. Surgical Therapy for Coronary Artery Disease among Patients With Combined Coronary Artery and Peripheral Vascular Disease. Circulation 1995; 91: 46-53.

5. M Bonacchi, F Battaglia, E Prifti, M Leacche, N S Nathan, G Sani et al. Early and late outcome of skeletonised bilateral internal mammary arteries anastomosed to the left coronary system. Heart 2005; 91: 195-202.

6. Andrew C, Don P, Charanjit SR, Bernard JG. The management of combined coronary arterydisease and peripheral vascular disease. Eur Heart J 2010; 31, 1565-1572. 